\title{
Prävention der kongenitalen Hüftdysplasie (developmental dysplasia of the hip DDH)*
}

\section{Max Geiser}

eingereicht am 17.5.2004

Korrespondenz: Prof. Dr. med. Max Geiser Gossetstrasse 49 CH-3084 Wabern
In der Diskussion über die Effektivität und die Kosten des sonographischen Screenings der Neugeborenenhüfte vermisse ich sowohl im Plädoyer des Gesundheitsökonomen [1] als auch im offenen Brief der Nationalen Ethikkommission an das Bundesamt für Gesundheit BAG [2] die Darstellung der bekannten Fakten über die DDH, der Geschichte der Bemühungen zur Verhütung der kongenitalen Hüftdysplasie und der Erfordernisse für ein erfolgreiches Screening einer Krankheit.

\section{Fakten zur «developmental dysplasia of the hip" DDH}

Das früher als angeborene Hüftluxation und Hüftdysplasie (congenital dislocation of the hip $\mathrm{CDH}$ ) bekannte und seit kurzem DDH genannte Krankheitsbild umfasst die Fehlentwicklung der Hüftgelenkpfanne (Acetabulum), die Instabilität (Subluxation und Luxation) und die teratologische Fehlartikulation. Ob die Instabilität zur Dysplasie des Acetabulums führt oder ob die Instabilität das Resultat der primär dysplastischen Entwicklung des Acetabulums ist, ist immer noch umstritten.

Für die neonatale Instabilität (Luxation und Luxierbarkeit) wird die Zahl von 5 bis 20 pro 1000 in der ersten Lebenswoche und von 1 bis 2 pro $1000 \mathrm{im}$ Alter von 3 Wochen genannt [3]. Für die sonographisch feststellbaren Abnormitäten werden in den ersten Lebenstagen Zahlen von weit über 100 pro 1000 genannt [4, 5], die bei der Kontrolle im Alter von 3 Wochen auf 4 pro 1000 absinkt [4]. Genetische Faktoren scheinen eine ursächliche Rolle für die Instabilität (Gelenkschlaffheit) und für die Tiefe des Acetabulums [6, 7] $\mathrm{zu}$ spielen. Postnatale Faktoren können die neonatale Instabilität verstärken. Die Hüftdysplasie ist bei den Lappen und kanadischen Indianern, die ihre Säuglinge mit gestreckten und adduzierten Hüften wickeln, häufiger als bei Chinesen und Afrikanern, die ihre Säuglinge mit abduzierten Beinen herumtragen. Bei den Bantu scheint die DDH unbekannt zu sein [8]. Steisslage begünstigt die Instabilität.

\section{Geschichte und Probleme der Präventionsbemühungen}

Vor 30 Jahren hat ein Präventionsenthusiast die unbefriedigende Situation bezüglich der Frühdiagnose und Behandlung der kongenitalen Hüftdysplasie in der Schweiz beanstandet [9] und darauf hingewiesen, dass die Bemühungen um die Frühdiagnose und Behandlung bereits 1929 mit dem orthopädischen Chirurgen Putti $[10,11]$ begannen, der die Herstellung einer Röntgenaufnahme des Beckens bei jedem Neugeborenen verlangte, um die Dysplasie der Pfanne als Vorstufe der Luxation erfassen zu können. Spätere Untersuchungen zeigten das Versagen der Röntgenuntersuchung bei der Darstellung des Knorpels und die Spontanheilung der voreilig radiologisch diagnostizierten Dysplasie des Acetabulums und die Gefahr der übereifrigen Behandlung [12].

Die Falscheinschätzung des Röntgenbildes, das erst mit der Ossifikation des Femurkopfes ( $a b$ 3. Monat) aussagekräftig wird, führte dazu, dass die Beobachtung von Ortolani 1935 [14], der das Repositionsschnappen der luxierten Hüfte bei der vorsichtigen Abduktion beschrieb, erst mehr als 20 Jahre später ernstgenommen wurde [15, 16]. Barlow unterschied zwischen der luxierten, reponierbaren und luxierbaren schlaffen Neugeborenenhüfte [16]. Von den von ihm in den ersten Tagen nach der Geburt untersuchten 5496 Kindern hatte im Alter von 12 Monaten kein einziges eine luxierte Hüfte, aber einige der wegen Instabilität behandelten Kinder zeigten Dysplasiezeichen der Pfanne. Die aus diesen Beobachtungen resultierende Ansicht, dass die Dysplasie nicht die Ursache, sondern die Folge der Instabilität sei, wurde durch genetische Untersuchungen $[6,17]$ in Frage gestellt, wonach sowohl die dominant vererbte Gelenkschlaffheit als auch die polygenetisch gesteuerte mangelhafte Entwicklung der Pfanne ätiologisch für die Instabilität des Gelenkes nach der Geburt und für die Schwierigkeiten der Erhaltung der Reposition eine Rolle spielen. Die Deutung der Instabilität als primäre Pathologie und als Ursache der dys- 
plastischen Entwicklung des Acetabulums führte zum Wechsel in der Nomenklatur von der «congenital dislocation of hip» $(\mathrm{CDH})$ zur «developmental dysplasia of the hip» (DDH). Bei diesem Nomenklaturwechsel wurde die polygenetisch vererbte dysplastische Pfannenentwicklung missachtet, die die später folgende Luxation von primär stabilen Hüftgelenken erklären könnte.

Nach den Erfolgsmeldungen aus Zentren mit einer grossen Zahl jährlicher Geburten mit konsequenter klinischer Untersuchung der Kinder kurz nach der Geburt auf Instabilität $[15,16]^{*}$ schien es zuerst, als ob das Problem der spätdiagnostizierten Dysplasiefälle mit notwendiger chirurgischer Behandlung (Reposition, Fixation, Tenotomie, blutige Reposition, Osteotomie) gelöst sei, bis nach einer Flut von Publikationen Zweifel an der Wirksamkeit des klinischen Screenings zur Elimination der spätdiagnostizierten Hüftluxation und Bedenken wegen der Folgen von Überbehandlung in Form von aseptischen Femurkopfnekrosen und Entwicklung einer Coxa vara geäussert [12, 13, 18-21] wurden.

\section{Die Bedeutung der Sonographie für die Prävention der DDH}

Die vor 20 Jahren für die Diagnose der DDH eingeführte Ultraschalluntersuchung [22] bietet die Möglichkeit, die anatomischen Verhältnisse der Neugeborenenhüfte vollständiger darzustellen als die für den Knorpel stummen Röntgenstrahlen. Aber die sowohl bei stabilen als auch instabilen Neugeborenenhüften festgestellten Abnormitäten der Tiefe der Pfanne und Überdachung des Femurkopfes sind so zahlreich [4, 5, 20-26], dass Überdiagnose, Überbehandlung und Interpretationsschwierigkeiten beanstandet, die Grafsche Klassifikation der Dysplasietypen als zu kompliziert [29] und als bedeutungslos [24] betrachtet wurden. Die Feststellung der Instabilität mit Hilfe der dynamischen Sonographie sei die einzige Technik von Bedeutung für die Entscheidung für oder wider eine Behandlung. Die Sonographie sei nicht imstande, Spätdiagnosen zu eliminieren [20, 21, 23]. Sie sei auf Risikofälle zu beschränken. Als Risiko für die Entwicklung einer Hüftdysplasie gelten klinisch festgestellte Instabilität, pos. Familiengeschichte, Steissgeburten, Kinder mit Deformitäten und Oligohydramnios. Die grösste Bedeutung hat die positive Familiengeschichte.

\section{Ergebnis der Präventionsbemühungen}

Die Literatur der Bemühungen zur Verbesserung der Behandlungsergebnisse der DDH ist auffallend kontrovers über die Wirksamkeit des klinischen und selektiven und universellen Ultraschallscreenings der Neugeborenenhüfte. Es herrscht lediglich Einigkeit darüber, dass es trotz den Präventionsbemühungen während 70 Jahren nicht gelungen ist, spätdiagnostizierte DDH, die eine Spitalbehandlung mit chirurgischen Eingriffen notwendig machen, zu vermeiden. Aber es steht fest, dass die Zahl dieser Kinder in den letzten 30 Jahren kleiner geworden ist $[4,18,20,27,28,30]$. Während sich in Deutschland und Österreich das universelle Ultraschallscreening etabliert hat, wird in Grossbritannien mit Ausnahme einer Gruppe in Coventry [4] das klinische Screening universell und die Sonographie selektiv nur für Risikofälle praktiziert, ohne mit den Ergebnissen zufrieden zu sein [23, 26, 30]. Der Bericht über die Ergebnisse des universellen Ultraschallscreenings in Deutschland [27] ist wissenschaftlich wegen methodischer Mängel fragwürdig. Denn mit Fragebogen und Telefoninterviews erhobene Daten sind unzuverlässig und lückenhaft besonders bezüglich der durch Überbehandlung entstehenden Schäden. Der Bericht aus Coventry [4] über drei verschiedene, gut dokumentierte Screeningmethoden während 20 Jahren zeigt einen Rückgang der Zahl der spätdiagnostizierten, eine Spitalbehandlung benötigenden Kinder bei der Ergänzung des klinischen Screenings durch Ultraschall für die Risikofälle und noch mehr nach der routinemässigen Durchführung der Sonographie durch erfahrene Radiologen und durch die Überwachung der erfassten Kinder. Bei sonographisch festgestellten Abnormitäten im Alter von 6 Wochen erfolgte unabhängig von der Stabilität die Behandlung mit der Pavlikbandage mit wöchentlichen Kontrollen bis zum Alter von 12 Wochen. Die bei der Sonographie festgestellten Abnormitäten sanken von 73 von 1000 Kindern kurz nach Geburt auf 4 pro 1000 nach 2-3 Wochen. Bei 0,6 pro 1000 war die Behandlung mit der Pavlikbandage nicht erfolgreich und erforderte eine geschlossene Reposition oder eine operative Behandlung. Wenn die Kosten für das Ultraschallscreening berücksichtigt werden, sind die Behandlungskosten bei klinischem Screening allein bei selektivem und universellem Sonographiescreening vergleichbar. Ein prospektiv randomisierter Test mit 15500 Säuglingen mit Vergleich des klinischen Screenings plus Ultraschall und des klinischen Screenings plus selektivem Ultraschall der Risikokinder [28] mit Überwachung während 6-11 Jahren zeigte keinen statistisch signifikanten Unterschied für spät entdeckte Dysplasien.

\footnotetext{
ren auch heutc ren wie z.B. in Grossbritannien.

Vor 30 Jahren war die Situation
bezüglich Frühdiagnose der kongenitalen Hüftdysplasie in der keine Morbiditätsstatistik. Bei 58 zwischen 1960 und 1968 geboreDaten einer Kammer der Invalition im Alter für diesen unbefriedigenden $\mathrm{Zu}$ stand wurde damit erklärt [9] holt untersucht werden und die Risikofälle nach der Spitalentlaswacht wurden. Ausserdem scheint die Zusammenarbeit zwischen Pädiatern und orthopädinoch nicht so gut zu funktionie-
} 


\section{Die Problematik des Screenings von Neugeborenenhüftgelenken}

Das Screening einer Krankheit ist sinnvoll, wenn diese häufig ist, ein verlässlicher Test für die Diagnose und eine verglichen mit dem natürlichen Krankheitsverlauf erfolgreiche Behandlungsmethode zur Verfügung steht und ökonomisch vertretbar ist. Diese Erfordernisse sind für das Screening der Neugeborenenhüfte nicht erfüllt, weil DDH selten ist $(0,65-1,5$ pro 1000$)$, ein verlässlicher diagnostischer Test und eine sichere Behandlungsmethode fehlt, die natürliche Entwicklung der Neugeborenenhüfte bis zum Gehbeginn nicht erforscht ist [18, 20, 23, 24], eine nicht risikofreie Überbehandlung droht, die Kompetenz und Erfahrung der untersuchenden Personen häufig zu wünschen übrig lässt und eine zuverlässige Kosten-Nutzen-Analyse fehlt. Es ist möglich, dass der Rückgang der Zahl der Spitalbehandlungen als Folge der DDH vor allem auf die schonendere nicht operative Behandlung und Überwachung der klinisch erfassten Risikokinder mit Vermeidung von Schäden durch Überbehandlung zurückzuführen ist.

\section{Schlussfolgerungen}

Die kongenitale Hüftdysplasie (developmental dysplasia of the hip DDH) ist trotz den Präventionsbemühungen während 70 Jahren eine ursächlich immer noch nicht geklärte Affektion. Die Entwicklung der unreifen Neugeborenenhüfte ist bis zum Gehbeginn (natural history) nicht hinreichend erforscht. Die Effektivität des klinischen Screenings und des selektiven und universellen Ultraschallscreenings ist nicht erwiesen. Das Screening wird unterschiedlich praktiziert. Die Wirksamkeit der Präventionsbemühungen scheint wesentlich von der Kompetenz, der Erfahrung und dem Engagement der Untersucher und einer lückenlosen Überwachung der Behandlung der Risikokinder durch erfahrene, zusammenarbeitende Pädiater, orthopädische Chirurgen und Radiologen abzuhängen. Überbehandlung und zu früher Behandlungsbeginn ist mit ernsthaften Schädigungen verbunden.

Guidelines für die Ultraschalluntersuchung der Neugeborenenhüfte bis zum Alter von $6 \mathrm{Mo-}$ naten wurden vom American College of Radiology in Zusammenarbeit mit dem American Institute of Ultrasound herausgegeben [30]. Die Indikationen für die besondere Überwachung der Neugeborenenhüfte sind festgelegt. Es handelt sich um die persistierende klinisch festgestellte Instabilität, die positive Familienge- schichte, die Steissgeburt und Deformitäten wie Schiefhals und Fussfehlstellungen und die sonographische Überwachung der Behandlung.

Solange die Effektivität des universellen Sonographiescreenings der Neugeborenenhüfte nicht erwiesen ist, kann eine Kosten-Nutzen-Diskussion [1] nicht in Betracht gezogen werden [31]. Ethische Überlegungen und Postulate [2] sind nicht spruchreif. Weitere Untersuchungen inklusive RCTs zur Klärung sinnvoller Präventionsmassnahmen liegen im Interesse künftiger Kinder und ihrer Eltern.

\section{Literatur}

1 Oggier W. Das Hüftsonographie-Screening - einige Gedanken eines Gesundheitsökonomen. Schweiz Ärztezeitung 2004;85:616-9.

2 Rehmann C. Sonographisches Hüftscreening bei Neugeborenen nach Graf. Offener Brief ans Bundesamt für Gesundheit. Schweiz Ärztezeitung 2004;85:620.

3 Salomon L, Warwick DJ, Nayagam S. Apley's System of Orthopaedics and Fractures. $8^{\text {th }}$ edition. London: Arnold; 2001.

4 Clegg J, Bache CE, Raut VV. Financial justification for routine ultrasound screening of the neonatal hip. J Bone Joint Surg Br 1999;81:852-7.

5 Tönnis D, Storck K, Ulblicht H. Results of newborn screening for congenital dislocation of hip with and without sonography and correlation of risk factors. J Pediatric Orthop 1990;10:145-52.

6 Wynne-Davies R. Acetabular dysplasia and familial joint laxity: two aetiological factors in congenital dislocation of the hip. J Bone Joint Surg Br 1970; 52:704-16.

7 Geiser M, Buri B, Buri P. Congenital dislocation of the hip in identical twins. J Bone Joint Surg Br 1959;41:314-8.

8 Edelstein J. Congenital dislocation of the hip in Bantu. J Bone Joint Surg 1966;48-B:397.

9 Geiser M. Frühdiagnose und Behandlung der kongenitalen Hüftluxation. Schweiz Med Wochenschr 1973;103:1449-54.

10 Putti V. Early treatment of congenital dislocation of the hip. J Bone Joint Surg 1929;11:798.

11 Putti V. Early treatment of congenital dislocation of the hip. J Bone Joint Surg 1933;15:16.

12 Geiser M. Dysplasie und Pseudodysplasie des kindlichen Hüftgelenkes. Z Orthop Ihre Grenzgeb 1971;115:1-8.

13 Geiser M. Behandlungsergebnisse bei frühzeitig diagnostizierter typischer congenitaler Hüftluxation. Z Orthop Ihre Grenzgebiete 1975;113:67-77.

14 Ortolani M. Un segno poco noto e la sua importanza per la diagnosi precoce dei prelussazione congenita degli anca. Pediatria (Napoli) 1935; 45:129. 
15 Andren M, von Rosen S. The diagnosis of dislocation of the hip in newborns and the primary results of immediate treatment. Acta Radiol 1958; 49:89-95.

16 Barlow TG. Early diagnosis and treatment of congenital dislocation of the hip. J Bone Joint Surg Br 1962;44:292-301.

17 Carter C, Wilkinson J. Persistent joint laxity and congenital dislocation of the hip. J Bone Joint Surg Br 1964;46:40-5.

18 Jones D. Neonatal detection of congenital dislocation of the hip (DDH). J Bone Joint Surg Br 1998; 80:943-5.

19 Boeree NR, Clarke NM. Ultrasound imaging and secondary screening for congenital dislocation of the hip. J Bone Joint Surg Br 1994;76:525-33.

20 Bennet GC. Screening for congenital dislocation of the hip. J Bone Joint Surg Br 1992;74:643-4.

21 Hernandez RJ, Cornell RG, Hensinger RN. Ultrasound diagnosis of neonatal congenital dislocation of the hip. J Bone Joint Surg Br 1994;76:539-43.

22 Graf R. New possibilities for the diagnosis of congenital hip joint dislocation by ultrasonography. J Pediatr Orthop 1983;3:354-9.

23 Paton RW, Srinivasan MS, Shah B, Hollis S. Ultrasound screening for hips at risk in developmental dysplasia. Is it worth it? J Bone Joint Surg Br 1999;81:255-8.
24 Engesaeter LB, Wilson DJ, Nag D, Benson MK. Ultrasound and congenital dislocation of the hip. J Bone Joint Surg Br 1990;72:197-201.

25 Danielson LG. Instability of the hip in neonates. J Bone Joint Surg Br 2000;82:545-7.

26 McKee L. Screening babies for hip dislocation is not effective. Br Med J 1998;346:1265.

27 von Kries R, Ihme N, Oberle D, Lorani A, Stark R, Altenhofen L, Niethard FU. Effect of ultrasound screening on the rate of first operative procedures for developmental hip dysplasia in Germany. Lancet 2003;362:1883-7.

28 Holen KJ, Tegnander A, Bredland T, Johansen OJ, Saether OD, Eik-Nes SH, Terjesen T. Universal or selective screening of the neonatal hip using ultrasound? J Bone Joint Surg Br 2002;84:886-90.

29 Marks DS, Clegg J, al-Chalabi AN. Routine ultrasound screening for neonatal hip instability. J Bone Joint Surg Br 1994;76:534-8.

30 American College of Radiology. Practice Guideline for the Performance of the Ultrasound Examination for Detection of Developmental Dysplasia of the Hip 1998, revised 2003, effective 1.10.2003.

31 Eastwood DM. Neonatal hip screening. Lancet 2003;361:595-7. 